УДК 616.895.8:616-003.682:615.015.6:616.8-008.64:616.89-008.441.44:303.625

Для цитирования: Климова И.Ю., Овчинников А.А., Карпушкин А.М. Выявление уровня депрессивного состояния и оценка риска суицидального поведения у больных шизофренией с коморбидной каннабиноидной зависимостью, сформировавшейся до манифестации шизофрении. Сибирский вестник психиатрии и наркологии. 2019; 4 (105): 67-73. https://doi.org/10.26617/1810-3111-2019-4(105)-67-73

\title{
Выявление уровня депрессивного состояния и оценка риска суицидального поведения у больных шизофренией с коморбидной каннабиноидной зависимостью, сформировавшейся до манифестации шизофрении
}

\author{
Климова И.Ю., Овчинников А.А., Карпушкин А.М.
}

Новосибирский государственный медииинский университет

Россия, 630091, Новосибирск, Красный проспект, 52

\section{PEЗЮME}

Цель: оценить степень выраженности депрессивного расстройства и возможный риск совершения суицида среди пациентов с установленным диагнозом «параноидная шизофрения», начавших употребление каннабиноидов до манифестации шизофрении, со стажем заболевания 3 года. Материал и методы. В данное исследование были включены 96 пациентов. Из них были сформированы две группы (по 48 человек в каждой группе). В основную (первую) группу вошли пациенты с установленным диагнозом «параноидная шизофрения», имеющие коморбидную каннабиноидную зависимость, с началом употребления каннабиноидов до манифестации шизофрении, срок длительности заболевания составлял не менее 3 лет. Контрольную (вторую) группу составили пациенты с установленным диагнозом «параноидная шизофрения», без зависимости от каннабиноидов, срок длительности заболевания у которых был не менее 3 лет. Средний возраст респондентов составил $23,19 \pm 1,74$ года. В работе использовались стуктурированное психиатрическое интервью для оценки психического статуса, социально-демографические сведения и клинико-катамнестический метод. Для оценки суицидального риска применялись шкала оценки суицидального риска TASR и опросник депрессии Бека. Результаты. Проведенное исследование показало, что лица, страдающие шизофренией, отягощенной коморбидной каннабиноидной зависимостью, более склонны к проявлению тяжелой депрессивной симптоматики и к суицидальному риску.

Ключевые слова: шизофрения, каннабиноидная зависимость, коморбидность, депрессивная симптоматика, суицидальный риск.

\section{АКТУАЛЬНОСТЬ}

В исследовании, проведенном в 2018 г. группой зарубежных исследователей из разных стран, объектами которого были подростки, проживающие в государствах с низким и средним уровнями доходов на душу населения, была установлена четкая связь между употреблением каннабиса и последующими суицидными попытками [1]. Метаанализ, проведенный итальянскими авторами, установил связь между употреблением препаратов каннабиса и последующей попыткой самоубийства у пациентов с биполярным аффективным расстройством [2]. Изучение 227 пар близнецов, выполненное австралийскими исследователями, показало, что риск суицидной попытки был в 2,9 раза выше для лиц, употребляющих каннабис [3]. Другие исследования, касающиеся лиц, страдающих каннабиноидной зависимостью, в свою очередь так же демонстрируют повышенную по сравнению с общей популяцией склонность таких лиц к суицидальному поведению $[3,4,5,6]$.
В литературе приводятся данные, что до $14 \%$ больных с коморбидной патологией склонны к повторению суицидальных попыток, из них 8,5\% носят завершенный характер. При этом законченные суициды характерны для пациентов мужского пола, в то время как женщины более склонны к совершению парасуицидов $[7,8,9,10]$. Однако имеющиеся исследования не отражают всей полноты проблемы, многие её аспекты трактуются противоречиво [7]. При этом разные аспекты изучения особенностей клинических проявлений и закономерностей формирования психических и поведенческих расстройств при злоупотреблении каннабиноидами остаются дискутабельными $[7,11,12]$.

Известно, что пациенты с двойным диагнозом имеют более низкие показатели социальноэкономического статуса и хуже функционируют в психосоциальном отношении. Такие больные труднее вовлекаются в лечебные программы и отличаются большей суицидоопасностью $[9,13,14]$. 
Частота суицидальных действий у больных шизофренией, коморбидной с аддиктивными расстройствами, по данным различных авторов, неодинакова и колеблется в обширных пределах - от 4,1 до 96\% [7, 10, 13, 15]. Суицидальные тенденции представляют собой одну из наиболее актуальных проблем, вызывающих серьезную озабоченность ученых и клиницистов-практиков, вследствие устойчиво сохраняющейся тенденции к увеличению частоты и выраженности коморбидной патологии.

\section{ЦЕЛЬ ИССЛЕДОВАНИЯ}

Оценка степени выраженности депрессивного расстройства и возможного риска суицида среди пациентов с установленным диагнозом «параноидная шизофрения», начавших употребление каннабиноидов до манифестации шизофрении), со стажем заболевания 3 года.

\section{МАТЕРИАЛЫ И МЕТОДЫ}

С учетом критериев отбора, после рандомизации в исследование были включены 96 пациентов. Все участники исследования были разделены на две группы. Основная группа (первая) включали 48 респондентов (24 женщины, 24 мужчины) с клинически установленным диагнозом «параноидная шизофрения» (рубрика F20.0 по МКБ-10) с коморбидной каннабиноидной зависимостью, с началом употребления каннабиноидов до манифестации шизофрении, причем длительность употребления каннабиноидов составляла не менее 3 лет (F12.0 по МКБ10). Контрольная (вторая) группа, так же как и основная группа, состояла из 48 респондентов (24 женщины, 24 мужчины) с клинически установленным диагнозом «параноидная шизофрения» (F20.0 по МКБ-10).

В работе использовалось информированное добровольное согласие на участие в исследовании. Все пациенты принимали адекватную психофармакотерапию. Доступность всех препаратов, включая атипичные нейролептики, была одинаковой. Возраст респондентов варьировал от 21 до 25 лет в обеих группах (средний возраст составил $23,19 \pm 1,74$ года).

Распределение обследуемых по социальнодемографическим характеристикам показало, что 94,05\% (79 человек) были одиноки; из них холостые - 81,1\%, разведенные - $18,9 \%$ $(\mathrm{p} \leq 0,05)$. Лишь $5,95 \%$ пациентов имели собственную семью. Большинство пациентов $(79,76 \%)$ оценили свои жилищные условия как достаточно благоприятные: в отдельных квартирах проживали 40,47\% респондентов, остальные проживали совместно с родственниками или родителями $(\mathrm{p} \leq 0,001)$.
На момент исследования все респонденты $(100 \%)$ не являлись инвалидами по какомулибо заболеванию. 90,5\% (76 человек) не были трудоустроены ( $\leq \leq 0,001)$, что может свидетельствовать о выраженной степени трудовой дезадаптации. Все обследуемые показали высокую степень критичности $(\mathrm{p} \leq 0,01)$ и практическое отсутствие дефицитарной симптоматики.

Исследование проводилось на фоне отсутствия острой психотической симптоматики более 2 месяцев, отсутствия интоксикации, вызванной употреблением каннабиноидов и других психоактивных веществ (ПАВ), отсутствия выраженного интеллектуально-мнестического снижения, отсутствия суицидальных попыток в анамнезе. В работе использовалось структурированное психиатрическое интервью для оценки психического статуса пациентов, социально-демографические и этнокультуральные сведения, клинико-катамнестический метод.

Для оценки суицидального риска были использованы шкала оценки суицидального риска (Tool for Assessment of Suicide Risk - TASR) и тест-опросник депрессии (Beck Depression Inventory - BDI).

Шкала TASR состоит из трех блоков: профиль индивидуального риска, включающий социально-демографические характеристики, профиль симптоматического риска и профиль риска на момент интервью. Суммарная оценка по данной шкале базируется на общем клиническом впечатлении в ходе обследования, в результате которого пациенты относятся к группам высокого, среднего или низкого суицидального риска [16].

Тест-опросник депрессии (Beck Depression Inventory), предложенный Аароном Т. Беком в 1961 г., включает в себя 21 вопросутверждение наиболее часто встречаемых симптомов и жалоб, соответствующих специфическим проявлениям/симптомам депрессии.

Статистическая обработка полученных результатов осуществлялась с применением пакета анализа Microsoft Exel. Достоверность различий определялась по t-критерию Стьюдента.

\section{РЕЗУЛЬТАТЫ ИССЛЕДОВАНИЯ}

В основной (первой) группе, включающей 48 респондентов, $7(14,5 \% ; \mathrm{p} \leq 0,005)$ человек набрали от 7 до 10 баллов, что говорит об очень высоком риске суицида; 12 человек $(25,0 \%$; $\mathrm{p} \leq 0,005)$ набрали 5-6 баллов, что интерпретируется как высокий риск суицида; 18 человек $(37,5 \% ; p \leq 0,005)$ набрали 3-4 балла, что свидетельствует о среднем риске суицида. Лишь 11 
человек $(23 \% ; \mathrm{p} \leq 0,005)$ показали низкий риск суицида, набрав от 0 до 2 баллов по шкале оценки суицидального риска (TASR).

B контрольной (второй) группе, так же состоящей из 48 респондентов, у одного пациента $(2,0 \% ; \mathrm{p} \leq 0,005)$ был выявлен очень высокий риск суицида; 2 пациента $(4,1 \% ; \mathrm{p} \leq 0,005)$ набрали 5-6 баллов, что соответствует высокому риску суицида; 8 человек $(16,6 \% ; \mathrm{p} \leq 0,005)$ набрали 3-4 балла, что трактуется как средний риск суицида. Преобладающее большинство пациентов (37 - 77,3\%; $\leq \leq 0,005)$ набрали 0-2 балла, что соответствует низкому риску суицид

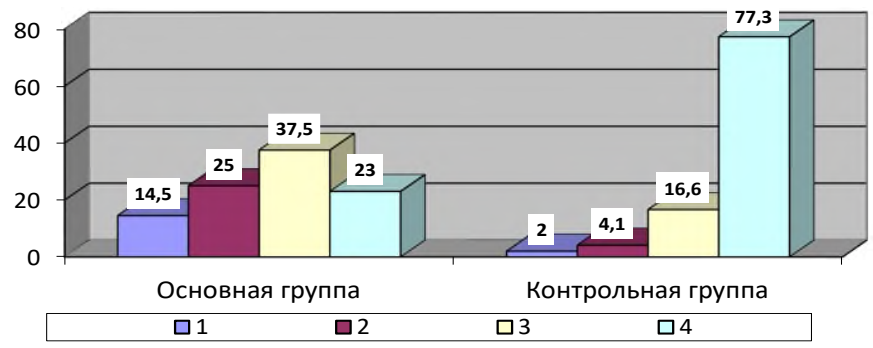

П р и м е ч а н и е. Риск суицида: 1 - очень высокий, 2 - высокий, 3 - средний, 4 - низкий.

Р и с у н о к 1. Соотношение суицидального риска по шкале оценки суицидального риска (TASR) в основной (первой) и контрольной (второй) группах исследования, \%

Полученные результаты по шкале депрессии Бека (BDI) продемонстрировали, что все испытуемые в обеих группах набрали более 10 баллов, что свидетельствует о наличии депрессивной симптоматики $(\mathrm{p} \leq 0,001)$. Так, в основной (первой) группе 12 человек $(25,0 \% ; \mathrm{p} \leq 0,005)$ набрали от 10 до 15 баллов, что свидетельствует о наличии легкой депрессии; 19 человек $(39,6 \% ; \mathrm{p} \leq 0,005)$ набрали 16-19 баллов, что оценивалось как умеренная депрессия; 17 человек $(35,4 \% ; \mathrm{p} \leq 0,005)$ - 20-29 баллов, что говорило о выраженной депрессии. Во второй (контрольной) группе данных за выраженную депрессию не обнаружено, у 37 человек $(77,1 \%$; $\mathrm{p} \leq 0,005)$ выявлена легкая депрессия, у 11 человек $(22,9 \% \mathrm{p} \leq 0,005)$ - умеренная депрессия согласно результатам тест-опросника депрессии (Beck Depression Inventory) (рис. 2).

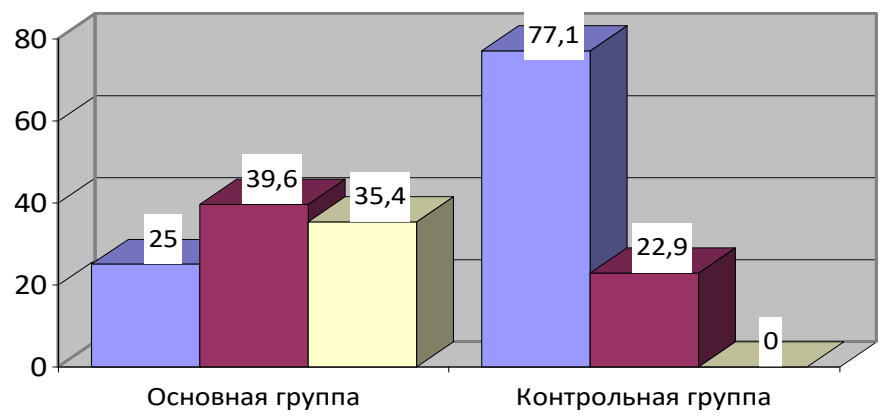

$\square$ Легкая депрессия

$\square$ Умеренная депрессия

$\square$ Выраженная депрессия

Р и с у н о к 2. Соотношение выраженности депрессивной симптоматики по шкале депрессии Бека (BDI) в основной (первой) и контрольной (второй) группах исследования 
В целом среди пациентов основной (первой) группы (пациенты с установленным диагнозом «параноидная шизофрения», начавшие употребление каннабиноидов до манифестации шизофрении, со стажем заболевания более 3 лет) в клинической картине депрессивного расстройства превалировали симптомы в виде выраженной физической и психической утомляемости, нарушения сердечного ритма в виде аритмий и удлинения интервала QT по данным ЭКГ, сухости во рту, запоров, нарушения менструального цикла у женщин и снижения потенции у мужчин. Данная симптоматика зачастую продолжительное время расценивалась как результат побочного эффекта терапии нейролептиками, в связи с чем прием антидепрессантов носил отсроченный характер. Нарушения сна, отмечавшиеся у большинства обследованных $(81,0 \%$; $\leq \leq 0,005)$, характеризовались частыми кошмарными сновидениями с выраженным эмоциональным компонентом, сопровождающимися ночными пробуждениями и продолжительным отсутствием сна или полным пробуждением и невозможностью уснуть до утра. Кроме того, более чем у половины обследованных $(67,0 \% ; \mathrm{p} \leq 0,005)$ отмечались тревога, беспричинное беспокойство, неуверенность в будущем. Менее чем у половины обследуемых $(43,0 \% ; p \leq 0,005)$ отмечались такие состояния, как невозможность долгое время находиться (усидеть) на одном месте (данное состояние зачастую расценивалось как проявление нейролептического синдрома), неконтролируемые движения (перебирают пальцами рук, кусают ногти. Для преобладающего большинства $(76,0 \% ; p \leq 0,005)$ пациентов характерна быстрая смена настроения - раздражительность легко сменяется плаксивостью.

В основной (первой) группе у 14,5\% обследованных выявлен очень высокий риск суицида; в $25,0 \%$ случаев - высокий риск суицида по шкале оценки суицидального риска (TASR). Полученные данные свидетельствуют о том, что пациенты основной группы в большей степени склонны к аффективным проявлениям депрессивного спектра с выраженной склонностью к суицидальным реакциям.

\section{ВЫВОДЫ}

Проведенное исследование показало, что среди пациентов, страдающих шизофренией, ассоциированной с синдромом каннабиноидной зависимости и начавших употребление каннабиноидов до манифестации шизофрении, в $39,6 \%(p \leq 0,005)$ случаев установлена умерен- ная депрессия; в $35,4 \%$ случаев отмечается выраженная депрессия согласно результатам тестопросника депрессии (Beck Depression Inventory). По шкале оценки суицидального риска (TASR) у $14,5 \% \quad(\mathrm{p} \leq 0,005)$ выявлен очень высокий риск суицида, у $25 \%(\mathrm{p} \leq 0,005)-$ высокий риск суицида.

Таким образом, употребление каннабиноидов до манифестации параноидной шизофрении является усугубляющим фактором в плане развития, формирования и течения ассоциированной депрессивной симптоматики, повышающей риск суицидального поведения.

\section{КОНФЛИКТ ИНТЕРЕСОВ}

Авторы заявляют об отсутствии явных и потенциальных конфликтов интересов в связи с публикацией данной статьи.

\section{ИСТОЧНИК ФИНАНСИРОВАНИЯ}

Авторы заявляют об отсутствии финансирования при проведении исследования.

\section{СООТВЕТСТВИЕ ПРИНЦИПАМ ЭТИКИ}

Работа соответствует нормам современных этических стандартов, разработанных в соответствии с Хельсинской декларацией ВМА.

\section{ЛИТЕРАТУРА}

1. Carvalho A.F., Stubbs B., Vancampfort D., Kloiber S., Maes M., Firth J., Koyanagi A. Cannabis use and suicide attempts among 86,254 adolescents aged 12-15 years from 21 low- and middle-income countries. European Psychiatry. 2019 Feb; 56: 8-13. doi: 10.1016/j.eurpsy.2018.10.006.

2. Bartoli F., Crocamo C., Carrà G. Cannabis use disorder and suicide attempts in bipolar disorder: A meta-analysis. Neuroscience \& Biobehavioral Reviews. 2019 Aug; 103: 14-20. doi: 10.1016/j.neubiorev.2019.05.017

3. Lynskey M.T., Glowinski A.L., Todorov A.A., Bucholz K.K., Madden P.A., Nelson E.C., Statham D.J., Martin N.G., Heath A.C. Major depressive disorder, suicidal ideation, and suicide attempt in twins discordant for cannabis dependence and early-onset cannabis use. Arch Gen Psychiatry. 2004 Oct; $\quad 61(10)$ : 1026-32. doi: 10.1001/archpsyc.61.10.1026

4. Delforterie M.J., Lynskey M.T., Huizink A.C., Creemers H.E., Grant J.D., Few L.R., Glowinski A.L., Statham D.J., Trull T.J., Bucholz K.K., Madden P.A., Martin N.G., Heath A.C., Agrawal A. The relationship between cannabis involvement and suicidal thoughts and behaviors. Drug Alcohol Depend. 2015 May 1; 150: 98-104. doi: 10.1016/j.drugalcdep.2015.02.019

5. Fergusson D.M., Woodward L.J., Horwood L.J. Risk factors and life processes associated with the onset of suicidal behavior during adolescence and early adulthood. Psychol Med. 2000 Jan; 30(1): 2339. doi: 10.1017/s003329179900135x 
6. Pedersen W. Does cannabis use lead to depression and suicidal behaviours? A population-based longitudinal study. Acta Psychiatr Scand. 2008 Nov; 118(5): 395-403. doi: 10.1111/j.16000447.2008.01259.x

7. Говорин Н.В., Сахаров А.В. Суицидальное поведение: типология и факторная обусловленность. Чита : Изд-во «Иван Федоров», 2008: 178.

8. Солохин Е.В., Каниболоцкий А.А., Чернолихова И.А., Лаптева М.И., Потемкин А.М. Отравление опиатами (анализ секционного материала). $C y$ дебно-медииинская экспертиза. 2002; 2: 32-35.

9. Сливко К.Ю. Клиническая классификация нефатальных суицидентов: дис... к.м.н. Владивосток, 2003: 160.

10. Шигеев С.В. Медико-социальные аспекты смертельных отравлений наркотическими веществами. Вопросы наркологии. 2006; 2: 68-72.

11. Корнетова Е.Г., Дмитриева Е.Г., Дубровская В.В., Меднова И.А., Гончарова А.А., Корнетов А.Н., Семке А.В., Иванова С.А., Бохан Н.А. Суицидальное поведение больных шизофренией с метаболическим синдромом. Суицидология. 2019; 10(2): 92-98.
12. Лапицкий М.А., Ваулин С.В. Суицидальное поведение. Смоленск: Изд-во СГМА МЗ РФ, 2000: 156.

13. Дудин И.И. Зависимость от каннабиноидов в структуре сочетанной психической патологии по данным отдаленного катамнеза (клинический, клинико-эпидемиологический, социодемографический аспекты): дис. ...д.м.н. М., 2009: 347.

14. Stillion J.M., McDouwell E.E. Suicide across the life span. Washington, Bristol, London: Taylor \& Francis, 1996: 349

15. Зотов П.Б., Бохан Н.А., Хохлов М.С., Петров И.М., Скрябин Е.Г., Зотова О.П., Петров В.Г. Суицидальные действия наркозависимых: вопросы системного выявления и учёта. Суицидология. 2019; 10, 1(34): 91-104. doi: 10.32878/suiciderus.19-10-01(34)-91-104

16. Kutcher S., Chehil S. Suicide risk management: A manual for health professionals. Malden, MA: Blackwell Publishing Ltd., 2007: 243.

\section{Поступила в редакцию 09.09.2019} Утверждена к печати 02.12.2019

Климова Ирина Юрьевна, ассистент кафедры психиатрии, наркологии и психотерапии ФГБОУ ВО НГМУ Минздрава России.

Овчинников Анатолий Александрович, доктор медицинских наук, профессор, заведующий кафедрой психиатрии, наркологии и психотерапии ФГБОУ ВО НГМУ Минздрава России.

Карпушкин Александр Михайлович, ассистент кафедры психиатрии, наркологии и психотерапии ФГБОУ ВО НГМУ Минздрава России.

Климова Ирина Юрьевна, iri.c2012@yandex.ru

УДК 616.895.8:616-003.682:615.015.6:616.8-008.64:616.89-008.441.44:303.625

For citation: Klimova I.Yu., Ovchinnikov A.A., Karpushkin A.M. Identification of the level of depressive state and evaluation of risk for suicidal behavior in patients with schizophrenia with comorbid cannabinoid dependence, having formed before the manifestation of schizophrenia. Siberian Herald of Psychiatry and Addiction Psychiatry. 2019; 4 (105): 67-73. https://doi.org/10.26617/1810-3111-2019-4(105)-67-73

\section{Identification of the level of depressive state and evaluation of risk for suicidal behavior in patients with schizophrenia with comorbid cannabinoid dependence, having formed before the manifestation of schizophrenia}

\section{Klimova I.Yu., Ovchinnikov A.A., Karpushkin A.M.}

Novosibirsk State Medical University

Krasnyy Avenue 52, 630091, Novosibirsk, Russian Federation

\section{ABSTRACT}

Objective: to evaluate the severity of depressive disorder and the possible risk for suicide among patients with a diagnosis of "paranoid schizophrenia" who started using cannabinoids before the manifestation of schizophrenia, with a 3-year history of the disease. Material and methods. 96 patients were enrolled in that study. Of these, two groups were formed (48 persons in each group). The main (first) group included patients with a diagnosis of "paranoid schizophrenia" who had comorbid cannabinoid dependence, with the start of cannabinoid use before the manifestation of schizophrenia, the length of the disease was at least 3 years. The control (second) group consisted of patients with a 
diagnosis of "paranoid schizophrenia", without dependence on cannabinoids, whose disease length was at least 3 years. The average age of the respondents was $23.19 \pm 1.74$ years. The study used a structured psychiatric interview to evaluate mental status, socio-demographic information, and a clinical follow-up method. To evaluate suicide risk, Tool for Assessment of Suicide Risk - TASR and Beck Depression Questionnaire were used. Results. The study showed that people with schizophrenia, complicated by comorbid cannabinoid dependence, are more prone to severe depressive symptoms and suicide risk.

Keywords: schizophrenia, cannabinoid dependence, comorbidity, depressive symptoms, suicide risk.

\section{REFERENCES}

1. Carvalho A.F., Stubbs B., Vancampfort D., Kloiber S., Maes M., Firth J., Koyanagi A. Cannabis use and suicide attempts among 86,254 adolescents aged 12-15 years from 21 low- and middle-income countries. European Psychiatry. 2019 Feb; 56: 8-13. doi: 10.1016/j.eurpsy.2018.10.006.

2. Bartoli F., Crocamo C., Carrà G. Cannabis use disorder and suicide attempts in bipolar disorder: A meta-analysis. Neuroscience \& Biobehavioral Reviews. 2019 Aug; 103: 14-20. doi: 10.1016/j.neubiorev.2019.05.017

3. Lynskey M.T., Glowinski A.L., Todorov A.A., Bucholz K.K., Madden P.A., Nelson E.C., Statham D.J., Martin N.G., Heath A.C. Major depressive disorder, suicidal ideation, and suicide attempt in twins discordant for cannabis dependence and early-onset cannabis use. Arch Gen Psychiatry. 2004 Oct; 61(10): 1026-32. doi: 10.1001/archpsyc.61.10.1026

4. Delforterie M.J., Lynskey M.T., Huizink A.C., Creemers H.E., Grant J.D., Few L.R., Glowinski A.L., Statham D.J., Trull T.J., Bucholz K.K., Madden P.A., Martin N.G., Heath A.C., Agrawal A. The relationship between cannabis involvement and suicidal thoughts and behaviors. Drug Alcohol Depend. 2015 May 1; 150: 98-104. doi: 10.1016/j.drugalcdep.2015.02.019

5. Fergusson D.M., Woodward L.J., Horwood L.J. Risk factors and life processes associated with the onset of suicidal behavior during adolescence and early adulthood. Psychol Med. 2000 Jan; 30(1): 2339. doi: 10.1017/s003329179900135x

6. Pedersen W. Does cannabis use lead to depression and suicidal behaviours? A population-based longitudinal study. Acta Psychiatr Scand. 2008 Nov; 118(5): 395-403. doi: 10.1111/j.16000447.2008.01259.x

7. Govorin N.V., Sakharov A.V. Suitsidal'noye povedeniye: tipologiya i faktornaya obuslovlennost' [Suicidal behavior: typology and factor dependence]. Chita: Publishing House "Ivan Fedorov", 2008: 178 (in Russian).

8. Solokhin E.V., Kanibolotsky A.A., Chernolikhova I.A., Lapteva M.I., Potemkin A.M. Otravleniye opiatami (analiz sektsionnogo materiala) [Opiate poisoning (analysis of sectional material)]. Sudebno-meditsinskaya ekspertiza - ForensicMedical Examination. 2002; 2: 32-35 (in Russian).
9. Slivko K.Yu. Klinicheskaya klassifikatsiya nefatal'nykh suitsidentov: dissertatsiya kandidata meditsinskikh nauk [Clinical classification of nonfatal suicides: PhD thesis]. Vladivostok, 2003: 160 (in Russian).

10. Shigeev S.V. Mediko-sotsial'nyye aspekty smertel'nykh otravleniy narkoticheskimi veshchestvami [Medical and social aspects of fatal drug poisoning]. Voprosy narkologii - Addiction Issues. 2006; 2: 68-72 (in Russian).

11. Kornetova E.G., Dmitrieva E.G., Dubrovskaya V.V., Mednova I.A., Goncharova A.A., Kornetov A.N., Semke A.V., Ivanova S.A., Bokhan N.A. Suitsidal'noye povedeniye bol'nykh shizofreniyey s metabolicheskim sindromom [Suicidal behavior of patients with schizophrenia with metabolic syndrome]. Suitsidologiya - Suicidology. 2019; 10(2): 92-98 (in Russian).

12. Lapitskiy M.A., Vaulin S.V. Suitsidal'noye povedeniye [Suicidal behavior]. Smolensk: Publishing House of the Smolensk State Medical Academy of the Ministry of Health of the Russian Federation, 2000: 156 (in Russian).

13. Dudin I.I. Zavisimost' ot kannabinoidov v strukture sochetannoy psikhicheskoy patologii po dannym otdalennogo katamneza (klinicheskiy, klinikoepidemiologicheskiy, sotsio-demograficheskiy aspekty): dissertatsiya doktora meditsinskikh nauk [Dependence on cannabinoids in the structure of combined mental pathology according to long-term follow-up data (clinical, clinical, epidemiological, socio-demographic aspects): MD thesis]. Moscow, 2009: 347 (in Russian).

14. Stillion J.M., McDouwell E.E. Suicide across the life span. Washington, Bristol, London: Taylor \& Francis, 1996: 349.

15. Zotov P.B., Bokhan N.A., Khokhlov M.S., Petrov I.M., Scriabin E.G., Zotova O.P., Petrov V.G. Suitsidal'nyye deystviya narkozavisimykh: voprosy sistemnogo vyyavleniya i uchota [Suicidal actions of drug addicts: issues of systemic identification and accounting]. Suitsidologiya - Suicidology. 2019; 10, 1(34): 91-104 (in Russian). doi: 10.32878/suiciderus.19-10-01(34)-91-104

16. Kutcher S., Chehil S. Suicide risk management: A manual for health professionals. Malden, MA: Blackwell Publishing Ltd., 2007: 243.

Received September 09.2019 Accepted December 02.2019

Klimova Irina Y., assistant of the Department of Psychiatry, Narcology and Psychotherapy, Novosibirsk State Medi- 
cal University, Novosibirsk, Russian Federation.

Ovchinnikov Anatoly A., MD, Prof., Head of the Department of Psychiatry, Narcology and Psychotherapy, Novosibirsk State Medical University, Novosibirsk, Russian Federation.

Karpushkin Alexander M., assistant of the Department of Psychiatry, Narcology and Psychotherapy, Novosibirsk State Medical University, Novosibirsk, Russian Federation.

$\triangle$ Klimova Irina Y., iri.c2012@yandex.ru 